Korean J. Math. 20 (2012), No. 2, pp. 263-271

\title{
A BIFURCATION PROBLEM FOR THE BIHARMONIC OPERATOR
}

\author{
Tacksun Jung And Q-Heung Choi*
}

\begin{abstract}
We investigate the number of the solutions for the biharmonic boundary value problem with a variable coefficient nonlinear term. We get a theorem which shows the existence of $m$ weak solutions for the biharmonic problem with variable coefficient. We obtain this result by using the critical point theory induced from the invariant function and invariant linear subspace.
\end{abstract}

\section{Introduction}

Let $\Omega$ be a bounded domain in $R^{n}$ with smooth boundary $\partial \Omega$. Let $\Delta$ be the elliptic operator and $\Delta^{2}$ be the biharmonic operator. Let $c \in R, a: \bar{\Omega} \rightarrow R$ be a continuous function and $g: \bar{\Omega} \rightarrow R$ be a $C^{1}$ function. Assume that $a(x)>0$ in $\bar{\Omega}$. In this paper we investigate the multiplicity of the weak solutions for the following variable coefficient nonlinear biharmonic equation with Dirichlet boundary condition

$$
\begin{gathered}
\Delta^{2} u+c \Delta u=\Lambda(a(x) u+g(u)) \quad \text { in } \Omega, \\
u=0, \quad \Delta u=0 \quad \text { on } \partial \Omega .
\end{gathered}
$$

Let $\lambda_{j}, j \geq 0$ be the eigenvalues and $\phi_{j}, j \geq 1$ be the corresponding eigenfunctions suitably normalized with respect to $L^{2}(\Omega)$ inner product and each eigenvalue $\lambda_{j}$ is repeated as often as its multiplicity, of the eigenvalue problem

$$
\Delta u+\lambda u=0 \quad \text { in } \Omega,
$$

Received May 9, 2012. Revised June 13, 2012. Accepted June 15, 2012.

2010 Mathematics Subject Classification: 35J30, 35J40.

Key words and phrases: biharmonic boundary value problem, critical point theory, invariant function, invariant subspace, $(P . S .)_{c}$ condition, eigenvalue problem.

This work(Tacksun Jung) was supported by Basic Science Research Program through the National Research Foundation of Korea(NRF) funded by the Ministry of Education, Science and Technology (KRF-2011-0026920).

${ }^{*}$ Corresponding author. 


$$
u=0 \quad \text { on } \partial \Omega \text {. }
$$

The eigenvalue problem

$$
\begin{aligned}
& \Delta^{2} u+c \Delta u=\mu a(x) u \quad \text { in } \Omega, \\
& u=0, \quad \Delta u=0 \quad \text { on } \partial \Omega,
\end{aligned}
$$

has also infinitely many eigenvalues $\mu_{j}=\lambda_{j}\left(\lambda_{j}-c\right), j \geq 1$ and corresponding eigenfunctions $\psi_{j}, j \geq 1$. We note that $\mu_{1}<\mu_{2} \leq \mu_{3} \ldots$, $\mu_{j} \rightarrow+\infty$.

We assume that $g$ satisfies the following conditions:

$(g 1) g \in C^{1}(R, R)$ and $g(\xi)=o(|\xi|)$ uniformly with respect to $x \in \bar{\Omega}$.

$(g 2) g(\xi)<0$ for any $\xi \in R$.

$(g 3) g(u)=-g(-u)$ for any $u \in \bar{\Omega}$.

Jung and Choi [4] showed the existence of at least two solutions, one of which is bounded solution and large norm solution of (1.1) when $g(u)$ is polynomial growth or exponential growth nonlinear term. The authors proved this result by the variational method and the mountain pass theorem. For the constant coefficient nonlinear case Choi and Jung [3] showed that the problem

$$
\begin{array}{cc}
\Delta^{2} u+c \Delta u=b u^{+}+s & \text { in } \Omega, \\
u=0, \quad \Delta u=0 \quad \text { on } \partial \Omega,
\end{array}
$$

has at least two nontrivial solutions when $\left(c<\lambda_{1}, \Lambda_{1}<b<\Lambda_{2}\right.$ and $s<0)$ or $\left(\lambda_{1}<c<\lambda_{2}, b<\Lambda_{1}\right.$ and $\left.s>0\right)$. The authors obtained these results by use of the variational reduction method. The authors [5] also proved that when $c<\lambda_{1}, \Lambda_{1}<b<\Lambda_{2}$ and $s<0$, (1.2) has at least three nontrivial solutions by use of the degree theory. Tarantello [9] also studied the problem

$$
\begin{gathered}
\Delta^{2} u+c \Delta u=b\left((u+1)^{+}-1\right) \quad \text { in } \Omega, \\
u=0, \quad \Delta u=0 \quad \text { on } \partial \Omega .
\end{gathered}
$$

She show that if $c<\lambda_{1}$ and $b \geq \Lambda_{1}$, then (1.3) has a negative solution. She obtained this result by the degree theory. Micheletti and Pistoia [7] also proved that if $c<\lambda_{1}$ and $b \geq \Lambda_{2}$, then (1.3) has at least four solutions by the vatiational linking theorem and Leray-Schauder degree 
theory. The authors [6] investigate the multiple solutions of semilinear elliptic equations. In this paper we are trying to find weak solutions of (1.1), that is,

$$
\int_{\Omega}\left[\Delta^{2} u \cdot v+c \Delta u \cdot v-\Lambda(a(x) u+g(u)) v\right] d x=0, \quad \forall v \in H,
$$

where $H$ is introduced in section 2 .

Our main result is the following.

Theorem 1.1. Let $\lambda_{j}<c<\lambda_{j+1}$. Assume that $a(x)>0$ and $g$ satisfies the conditions $(g 1)-g(3)$. If $\mu_{k}<\Lambda<\mu_{k+1}, k \geq j+1$, then (1.1) has at least $k$ weak solutions.

We prove Theorem 1.1 by the critical point theory induced from the invariant subspace and invariant functional. The outline of the proof of Theorem 1.1 is as follows: In section 2, we introduce a Hilbert space $H$ and a closed invariant linear subspace $X$ of $H$ which is invariant under the operator $u \mapsto \int_{\Omega}|\Delta u|^{2}-c|\nabla u|^{2} d x$, the invariant subspaces of $X$ and the invariant function on $X$. We obtain some results on the norm $\|\cdot\|$ and the functional $f(u)$, and recall a critical point theory in terms of the invariant functional and invariant subspaces which plays a crucial role for the proof of the main result. In section 3, we prove Theorem 1.1.

\section{Critical point theory induced from the invariant subspace and the invariant function}

Let $L^{2}(\Omega)$ be a square integrable function space defined on $\Omega$. Any element $u$ in $L^{2}(\Omega)$ can be written as

$$
u=\sum h_{k} \phi_{k} \quad \text { with } \sum h_{k}^{2}<\infty .
$$

We define a subspace $H$ of $L^{2}(\Omega)$ as follows

$$
H=\left\{u \in L^{2}(\Omega)\left|\sum\right| \mu_{k} \mid h_{k}^{2}<\infty\right\} .
$$

Then this is a complete normed space with a norm

$$
\|u\|=\left[\sum\left|\mu_{k}\right| h_{k}^{2}\right]^{\frac{1}{2}} .
$$

Since $\lambda_{k} \rightarrow+\infty$ and $c$ is fixed, we have 
(i) $\Delta^{2} u+c \Delta u \in H$ implies $u \in H$.

(ii) $\|u\| \geq C\|u\|_{L^{2}(\Omega)}$, for some $C>0$.

(iii) $\|u\|_{L^{2}(\Omega)}=0$ if and only if $\|u\|=0$, which is proved in [2].

Let

$$
\begin{aligned}
& H^{+}=\left\{u \in H \mid h_{k}=0 \text { if } \mu_{k}<0\right\}, \\
& H^{-}=\left\{u \in H \mid h_{k}=0 \text { if } \mu_{k}>0\right\} .
\end{aligned}
$$

Then $H=H^{-} \oplus H^{+}$, for $u \in H, u=u^{-}+u^{+} \in H^{-} \oplus H^{+}$. Let $P_{+}$be the orthogonal projection on $H^{+}$and $P_{-}$be the orthogonal projection on $H^{-}$. We can wtite $P_{+} u=u^{+}, P_{-} u=u^{-}$, for $u \in H$. We are looking for the weak solutions of (1.1). By the following Proposition 2.1, the weak solutions of (1.1) coincide with the critical points of the associated functional

$$
\begin{gathered}
I(u) \in C^{1}(H, R), \\
(2.1) I(u)=\int_{\Omega}\left[\frac{1}{2}|\Delta u|^{2}-\frac{c}{2}|\nabla u|^{2}-\Lambda \int_{\Omega}\left[\frac{1}{2} a(x) u^{2}+G(u)\right] d x\right. \\
=\frac{1}{2}\left(\left\|P_{+} u\right\|^{2}-\left\|P_{-} u\right\|^{2}\right)-\Lambda \int_{\Omega}\left[\frac{1}{2} a(x) u^{2}+G(u)\right] d x,
\end{gathered}
$$

where $G(\xi)=\int_{0}^{\xi} g(\tau) \tau$. By $(g 1), I$ is well defined.

Proposition 2.1. Assume that $\lambda_{j}<c<\lambda_{j+1}, j \geq 1$, and $g$ satisfies $(g 1)-(g 3)$. Then $I(u)$ is continuous and Fréchet differentiable in $H$ with Fréchet derivative

$$
\nabla I(u) h=\int_{\Omega}[\Delta u \cdot \Delta h-c \nabla u \cdot \nabla h-\Lambda(a(x) u+g(u)) h] d x .
$$

If we set

$$
F(u)=\Lambda \int_{\Omega}\left[\frac{1}{2} a(x) u^{2}+G(u)\right] d x,
$$

then $F^{\prime}(u)$ is continuous with respect to weak convergence, $F^{\prime}(u)$ is compact, and

$$
F^{\prime}(u) h=\Lambda \int_{\Omega}(a(x) u+g(u)) h d x \quad \text { for all } h \in H,
$$

this implies that $I \in C^{1}(H, R)$ and $F(u)$ is weakly continuous. 
The proof of Proposition 2.1 has the similar process to that of the proof in Appendix $B$ in [8].

Let us define some notations and concepts on $Z_{2}$-invariant set and $Z_{2}$-invariant function: Let $H$ be a real Hilbert space on which the action $Z_{2}$ acts orthogonally. For $u \in H$, we define $Z_{2}-$ actions on $H$ by

$$
T u=u \quad \text { or } T u=-u \text {. }
$$

that is, the $Z_{2}$ action have the identity map and the antipodal map as an action. Thus $Z_{2}-$ action acts freely on the subspace $\{u \mid T u=-u\}$. Let $\operatorname{Fix}_{Z_{2}}$ be the set of fixed points of the action, i.e.,

$\operatorname{Fix}_{Z_{2}}=\left\{u \in H \mid T u(x)=u(x)\right.$, for all $\left.x \in \Omega, u \in H, Z_{2}-\operatorname{action} T\right\}$. We note that $\operatorname{Fix}_{Z_{2}}=\{0\}$. Let

$$
X_{1}=\operatorname{Fix}_{Z_{2}}=\{0\} \quad X_{2}=X_{1}^{\perp} .
$$

Thus $Z_{2}-$ action has the representation $x \mapsto-x$, for $x \in X_{2}$ and $H=$ $X_{1} \oplus X_{2}$. We say a subset $B$ of $H$ an $Z_{2}$-invariant set if for all $u \in B$, $T u \in B$. A function $I: H \rightarrow R^{1}$ is called $Z_{2}$-invariant if $I(T u)=I(u)$, $\forall u \in H$. Let $C(B, H)$ be the set of continuous functions from $B$ into $H$. If $B$ is an invariant set we say $h \in C(B, H)$ is an equivariant map if $h(T u)=T h(u)$ for all $u \in B$. We note that $H$ is a closed invariant linear subspace of $H$ compactly embedded in $L^{2}(\Omega, R)$ under the $Z_{2}-$ action. Let

$$
(L u) h=\int_{\Omega}[\Delta u \cdot \Delta h-c \nabla u \cdot \nabla h] d x .
$$

We can check easily that $L(H) \subseteq H, L: H \rightarrow H$ is an isomorphism and $\nabla I(H) \subseteq H$. Therefore constrained critical points on $H$ are in fact free critical points on $H$. Moreover, distinct critical orbits give rise to geometrically distinct solutions. We have the following lemma which can be checked easily since Fix $_{Z_{2}}=\{0\}$ :

Lemma 2.1. Assume that $g$ satisfies the conditions $(g 1)-(g 3)$. Let $u \in F_{i X_{Z_{2}}}=\{0\}$ and $u$ be a critical point of the functional of $I$, i.e., $\nabla I(u)=0$. Then $I(u)=0$.

Now we recall the critical point theory in terms of the invariant subspace and invariant function in Theorem 4.1 of [1] which plays a crucial role for the proof of Theorem 1.1: Let $S_{\rho}$ be the sphere centered at the origin of radius $\rho$. Let $I: H \rightarrow R$ be a functional of the form

$$
I(u)=\frac{1}{2}(L u) u-F(u),
$$


where $L: H \rightarrow H$ is linear, continuous, symmetric and equivariant, $F: H \rightarrow R$ is of class $C^{1}$ and invariant and $D F: H \rightarrow H$ is compact.

Theorem 2.1. Assume that $I \in C^{1}\left(H, R^{1}\right)$ is $Z_{2}$-invariant and there exist two closed invariant linear subspaces $V, W$ of $H$ and $\rho>0$ with the following properties:

(a) $V+W$ is closed and of finite codimension in $H$;

(b) $\operatorname{Fix}_{Z_{2}} \subseteq V+W$;

(c) $L(W) \subseteq W$;

(d) $\sup _{S_{\rho} \cap V} I<+\infty$ and $\inf _{W} I>-\infty$;

(e) $u \notin$ Fix $_{Z_{2}}$ whenever $D I(u)=0$ and $\inf _{W} I \leq I(u) \leq \sup _{S_{\rho} \cap V} I$;

(f) I satisfies $(P . S .)_{c}$ condition whenever $\inf _{W} I \leq c \leq \sup _{S_{\rho} \cap V} I$.

Then I possesses at least

$$
\operatorname{dim}(V \cap W)-\operatorname{codim}_{H}(V+W)
$$

distinct critical orbits in $I^{-1}\left(\left[\inf _{W} I, \sup _{S_{\rho} \cap V} I\right]\right)$.

\section{Proof of Theorem 1.1}

To prove Theorem 1.1 we shall prove that the functional $I$ satisfies the assumptions $(g 1)-g(3)$ of Theorem 2.1. We assume that $g$ satisfies the conditions $(g 1)-(g 3)$. Let us set

$$
\begin{gathered}
H_{1}^{+}=\left\{u \mid u \in H, u \in \operatorname{span}\left\{\psi_{l}, l \geq 1\right\}\right\}, \\
H_{k}^{-}=\left\{u \mid u \in H, u \in \operatorname{span}\left\{\psi_{l}, 1 \leq l \leq k\right\}\right\} .
\end{gathered}
$$

We have the following lemma which can be checked easily since Fix $Z_{Z_{2}}=$ $\{0\}$ :

Lemma 3.1. Assume that $g$ satisfies the conditions $(g 1)-(g 3)$. Then there exist $\rho>0$ and a sphere $S_{\rho}$ centered at 0 in $H$ such that the 
functional $I(u)$ is bounded from above on $S_{\rho} \cap H_{k}^{-}$and from below on $H_{1}^{+}$. That is,

$$
-\infty<\inf _{u \in H_{1}^{+}} I(u) \quad \text { and } \quad \sup _{u \in S_{\rho} \cap H_{k}^{-}} I(u)<0 .
$$

Proof. We note that

$$
\begin{aligned}
& \forall u \in H_{k}^{-}:(L u) u \leq \mu_{k} \int_{\Omega} a(x) u^{2} d x, \\
& \forall u \in H_{1}^{+}:(L u) u \geq \mu_{1} \int_{\Omega} a(x) u^{2} d x .
\end{aligned}
$$

Then for $u \in H_{k}^{-}$,

$$
\begin{aligned}
I(u) & =\frac{1}{2}(L u) u-\Lambda \int_{\Omega}\left[\frac{1}{2} a(x) u^{2}+G(u)\right] d x \\
& \leq \frac{1}{2}\left(\mu_{k}-\Lambda\right) \int_{\Omega} a(x) u^{2} d x+o\left(\|u\|_{L^{2}(\Omega)}^{2}\right)
\end{aligned}
$$

since $G(\xi) \in C^{2}$. Thus we can choose a number $\rho>0$ and a sphere $S_{\rho}$ centered at 0 in $H$ such that for any $u \in S_{\rho}$,

$$
\begin{aligned}
& \frac{1}{2}\left(\mu_{k}-\Lambda\right) \int_{\Omega} a(x) u^{2} d x+o\left(\|u\|_{L^{2}(\Omega)}^{2}\right) \\
& \leq \frac{1}{2}\left(\mu_{k}-\Lambda\right)(\sup a(x)) \rho^{2}+o\left(\|u\|_{L^{2}(\Omega)}^{2}\right)<0
\end{aligned}
$$

since $\mu_{k}-\Lambda<0$. Thus we have $\sup _{S_{\rho} \cap H_{k}^{-}} I(u)<0$. Let $u \in H_{1}^{+}$. Then we have

$$
\begin{aligned}
I(u) & =\frac{1}{2}(L u) u-\Lambda \int_{\Omega}\left[\frac{1}{2} a(x) u^{2}+G(u)\right] d x \\
& \geq \frac{1}{2}\left(\mu_{1}-\Lambda\right) \int_{\Omega} a(x) u^{2} d x+o\left(\|u\|_{L^{2}(\Omega)}^{2}\right) \\
& >\frac{1}{2}\left(\mu_{1}-\Lambda\right)(\sup a(x))\|u\|_{L^{2}}^{2}+o\left(\|u\|_{L^{2}(\Omega)}^{2}\right) \\
& >-\infty
\end{aligned}
$$

since $\mu_{1}-\Lambda<0, G(u)=o\left(\|u\|_{L^{2}(\Omega)}^{2}\right)$.

Thus we have $\inf _{u \in H_{1}^{+}} I(u)>-\infty$.

Lemma 3.2. Assume that $g$ satisfies the conditions $(g 1)-(g 3)$. Then the functional I satisfies $(P . S .)_{c}$ condition for every $c \in\left[\inf _{W} I(u), \sup _{S_{\rho} \cap V} I(u)\right]$. 
Proof. Let $u \in H$. Since $H=H_{1}^{+}$, the functional

$$
\begin{aligned}
I(u) & =\frac{1}{2}(L u) u-\Lambda \int_{\Omega}\left[\frac{1}{2} a(x) u^{2}+G(u)\right] d x \\
& \geq \frac{1}{2}\left(\mu_{1}-\Lambda\right) \int_{\Omega} a(x) u^{2} d x-\Lambda \int_{\Omega} G(u) d x \\
& >\frac{1}{2}\left(\mu_{1}-\Lambda\right) \sup (a(x))\|u\|_{L^{2}}^{2}-o\left(\|u\|_{L^{2}}^{2}\right) \\
& \geq \frac{1}{2}\left(\mu_{1}-\Lambda\right) \sup (a(x))\|u\|_{L(\Omega)}^{2}-o\left(\|u\|_{L^{2}}^{2}\right) \\
& >-\infty .
\end{aligned}
$$

Thus $I(u)$ is bounded from below since $G(\xi)=o\left(|\xi|^{2}\right)$. Thus $I(u)$ satisfies the $(P . S .)_{c}$ condition.

[Proof of Theorem 1.1]

If we set $V=H_{k}^{-}$and $W=H_{1}^{+}=H$, then $V+W$ is closed invariant subspaces of $H$ with $V+W=H$ and of finite codimension in $H$. We note that $\operatorname{Fix}_{Z_{2}}=\{0\}$ and $\operatorname{Fix}_{Z_{2}}=\{0\} \subseteq V+W=H$. We also note that $L(W) \subseteq W$. By Lemma 3.1,

$$
-\infty<\inf _{W} I \quad \sup _{H_{k}^{-}} \cap S_{\rho} I<0 .
$$

Thus the condition (d) of Theorem 2.1 is satisfied. Suppose that $u$ be a critical point of the functional of $I$ and $\inf _{W} I \leq I(u) \leq \sup _{S_{\rho} \cap V} I$. Then by Lemma 3.1, $-\infty<\inf _{W} I \leq I(u) \leq \sup _{S_{\rho} \cap V} I<0$. We claim that $u \notin \operatorname{Fix}_{Z_{2}}$. If not, then $u \in \operatorname{Fix}_{Z_{2}}=\{0\}$ i.e., $u=0$. Since $u=0$ is a critcal point of $I(u)$ with $I(0)=0$ and $0 \notin\left[\inf _{W} I, \sup _{S_{\rho} \cap V} I\right]$, it leads to a contradiction to the fact that $\inf _{W} I \leq I(u) \leq \sup _{S_{\rho} \cap V} I$. Thus $u \notin \operatorname{Fix}_{Z_{2}}$. Thus the condition $(e)$ is satisfied. By Lemma 3.2, I satisfies $(P . S .)_{c}$ condition whenever $\inf _{W} I \leq c \leq \sup _{S_{\rho} \cap V} I$.

Thus the assumptions $(a)-(e)$ of Theorem 1.1 are satisfied. Thus by the Theorem 2.1, Then $I$ possesses at least

$$
\operatorname{dim}(V \cap W)-\operatorname{codim}_{H}(V+W)=k
$$

distinct critical orbits in $I^{-1}\left(\left[\inf _{W} I, \sup _{S_{\rho} \cap V} I\right]\right)$. 


\section{References}

[1] K.C. Chang, Infinite dimensional Morse theory and multiple solution problems, Birkhäuser, (1993).

[2] Q.H. Choi, T.S. Jung, Multiplicity of solutions and source terms in a fourth order nonlinear elliptic equation, Acta Math. Sci. 19 (4) (1999), 141-164.

[3] Q.H. Choi, T.S. Jung, Multiplicity results on nonlinear biharmonic operator, Rocky Mountain J. Math. 29 (1) (1999), 141-164.

[4] T.S. Jung, Q.H. Choi, Nonlinear biharmonic problem with variable coefficient exponential growth term, Korean J. Math., 18 (3) (2010), 1-12.

[5] T.S. Jung, Q.H. Choi, Multiplicity results on a nonlinear biharmonic equation, Nonlinear Anal. 30 (8) (1997), 5083-5092.

[6] A.C. Lazer, J.P. Mckenna, Multiplicity results for a class of semilinear elliptic and parabolic boundary value problems, J. Math. Anal. Appl., 107 (1985), 371395.

[7] A.M. Micheletti, A. Pistoia, Multiplicity results for a fourth-order semilinear elliptic problem, Nonlinear Anal. 31 (7) (1998), 895-908.

[8] P.H. Rabinowitz, Minimax methods in critical point theory with applications to differential equations, CBMS Reg. Conf. Ser. Math., 65, Amer. Math. Soc., Providence, Rhode Island (1986).

[9] Tarantello, A note on a semilinear elliptic problem, Differential Integral Equations, 5 (3) (1992), No. 3, 561-565.

Department of Mathematics

Kunsan National University

Kunsan 573-701, Korea

E-mail: tsjung@kunsan.ac.kr

Department of Mathematics Education

Inha University

Incheon 402-751, Korea

E-mail: qheung@inha.ac.kr 